\title{
HIGH NUCLEAR PHOSPHORYLATED EXTRACELLULAR SIGNAL-REGULATED KINASE EXPRESSION ASSOCIATED WITH POOR DIFFERENTIATION, LARGER TUMOR SIZE, AND ADVANCED STAGE OF BREAST CANCER
}

\author{
Hsing-Tao KuO ${ }^{1 *}$, Hui-Ting Hsu ${ }^{2 *}$, Chun-Chao Chang ${ }^{3,4}$, Ming-Chung Jiang ${ }^{3}$, \\ Chung-Min Yeh ${ }^{2,5}$, Ko-Hung Shen ${ }^{2}$, Pei-Chi Hsu ${ }^{6}$, Cheng-Jeng Tai ${ }^{3,7}$
}

\begin{abstract}
*Dr. Kuo HT and Dr. Hsu HT contributed equally to this paper.
${ }^{1}$ Department of Internal Medicine, Division of Hepatogastroenterology, Chi-Mei Medical Center, Tainan, Taiwan 2Department of Pathology, Changhua Christian Hospital, Changhua, Taiwan

3Department of Internal Medicine, School of Medicine, College of Medicine, Taipei Medical University, Taipei, Taiwan 4Division of Gastroenterology and Hepatology, Department of Internal Medicine, Taipei Medical University Hospital, Taipei, Taiwan

${ }^{5}$ Department of Medical Technology, Jen-Teh Junior College of Medicine, Nursing and Management, Miaoli, Taiwan 6Institute of Cellular and Organismic Biology, Academia Sinica, Taipei, Taiwan

${ }^{7}$ Division of Hematology and Oncology, Department of Internal Medicine, Taipei Medical University Hospital, Taipei, Taiwan
\end{abstract}

\begin{abstract}
Extracellular signal-regulated kinase (ERK1/2) is implicated in the malignant behavior of breast cancer cells. However, previous clinical-pathological studies have shown that expression of activated/phosphorylated ERK1/2 is not associated with enhanced proliferation and invasion of mammary carcinomas. ERK1/2 is expressed in the cytoplasm, and activated/phosphorylated ERK1/2 translocates to the nucleus. The aim of this study is to evaluate nuclear phosphorylated ERK1/2 as a biomarker for breast cancer prognosis. The clinical-pathological relation of cytoplasmic/nuclear phosphorylated ERK1/2 was analyzed in 105 surgically resected breast cancer specimens by immunohistochemistry with tissue microarray. The results showed that non-neoplastic breast tissue mainly showed faint phosphorylated ERK1/2 staining. No statistically significant association was found between the level of cytoplasmic phosphorylated ERK1/2 expression and the clinical features of the disease. High nuclear phosphorylated ERK1/2 expression was associated with high grade (poor differentiation, $\mathrm{p}=0.010$ ), high $\mathrm{T}$ status (larger tumor size, $\mathrm{p}=0.033$ ), and an advanced stage $(p=0.018)$ of the disease. Thus, nuclear phosphorylated ERK1/2 is associated with enhanced proliferation and invasion of mammary carcinomas and may be a biomarker for breast cancer prognosis and the determination of therapeutic strategies.
\end{abstract}

Key words: breast cancer, ERK1/2, nuclear, phosphorylation, prognosis.

\section{Introduction}

Breast cancer is one of the most common cancers affecting women. Small and primary breast cancers are considered potentially curable by surgical operation.
However, poorly differentiated, high $\mathrm{T}$ status, advancedstage, and metastatic breast cancers require much more complex medical treatments [1-4]. The determination of appropriate therapeutic strategies plays an important role in reducing the mortality of the disease [5]. 
The identification of biomarkers associated with the clinical-pathological correlation of breast cancer can improve prognosis and aid in therapeutic strategy determination.

The extracellular signal-regulated kinase ERK1/2 plays an important role in mediating the transduction of extracellular signals to the nuclei of cells [6]. The ERK $1 / 2$ protein is expressed in cytoplasm, and the activation of ERK $1 / 2$ by its upstream signaling results in ERK1/2 phosphorylation [6]. Nuclear localization of phospho-ERK1/2 modulates gene transcription [7]. It has been reported that the phosphorylation and activation of ERK $1 / 2$ was disassociated from nuclear translocalization and transcription of downstream targets in primary cultures of breast and ovarian epithelial cells, indicating that the nuclear localization of phospho-ERK1/2 plays an important role in cancer development [8].

Aberrant ERK1/2 activation is common in cancer because of the mutational activation and overexpression of the upstream mitogen-activated protein kinase (MAPK) signaling pathway [9, 10]. Studies have shown that breast cancers frequently contain an increased proportion of cells with activated MAPK signaling [11, 12]. Additionally, studies have reported a trend toward higher MAPK signaling in the primary tumors of nodepositive patients than in the primary tumors of nodenegative patients [11]. It was reported recently that the levels of ERK1/2 expression were correlated with shorter survival in triple-negative breast cancer patients [13]. Studies have also shown that the expression of ERK1/2 was higher in breast cancer specimens, compared with control tissues, and was higher in Stage III patients than in Stage I and Stage II patients [14]. In addition, the expression of ERK $1 / 2$ in breast carcinoma tissue was lower in patients who had received preoperative chemotherapy of 5'-deoxy-5-fluorouridine (5'-DFUR), indicating that ERK $1 / 2$ plays a crucial role in the proliferation and development of breast cancer [14]. An in vitro study with extracellular signal-regulated kinase activator kinase (MEK1) inhibitors showed that ERK1/2 activity correlates with the proliferation and motility of breast cancer cells $[15,16]$. However, previous studies with clinical breast cancer samples and immunohistochemistry with antibodies against phospho-ERK1/2 have shown that activation of ERK1/2 was not associated with enhanced proliferation and invasion of mammary carcinomas [17].

ERK $1 / 2$ is expressed in the cytoplasm, and the activated/phosphorylated ERK1/2 translocates to the nucleus and is implicated in the invasion of tumor cells $[6,7,18]$. We investigated the cytoplasmic/nuclear expression pattern of phosphorylated ERK $1 / 2$ in breast tumors to evaluate nuclear phosphorylated ERK1/2 as a biomarker for breast cancer prognosis. Our results suggest that nuclear phosphorylated ERK plays an oncogenic role in breast cancer proliferation and progres- sion and may be a biomarker for the prognosis of breast cancer.

\section{Material and methods}

\section{Patients}

Breast tumor samples were obtained from 105 cases of breast cancer patients diagnosed with invasive ductal carcinoma. The tumors were graded and staged according to the Staging Manual ( $7^{\text {th }}$ ed.) of the American Joint Committee on Cancer [19]. The patients' mean age was 51.9 years (ranging from 29.8 to 80.9 years). Twenty-eight patients had Stage II tumors and 77 patients had Stage III tumors. No Stage I or Stage IV tumors were present in the samples. The study was approved by the Ethics Committees of Changhua Christian Hospital (Changhua, Taiwan) and adhered to the guidelines approved by the Institutional Review Board.

\section{Tissue microarrays and immunohistochemistry}

For tissue microarrays, three tissue cores from cancerous breast tissue and one core from cancer-free tissue in each paraffin block were longitudinally cut and arranged into new paraffin blocks using a manual method with the BiosynMatric Handmade Kit (Formosa Transcrip, Kaohsiung, Taiwan). The samples were used to generate tissue microarrays. Immunohistochemistry was performed on the tissue microarrays as previously described [20]. The tissue sections were deparaffinized in xylene and rehydrated in graded alcohol. Antigen retrieval was performed by treatment with a boiling citrate buffer ( $10 \mathrm{mmol} / \mathrm{l}, \mathrm{pH}$ 6.0) for $20 \mathrm{~min}$. Endogenous peroxidase activity was blocked using 3\% hydrogen peroxide in water, and nonspecific staining was blocked by incubation with $5 \%$ bovine serum albumin in phosphate buffered saline (PBS) for $1 \mathrm{~h}$ at room temperature. The samples were incubated with a 200-fold dilution of anti-phospho-ERK1/2 antibodies (phospho T202/204, G15-B) (Abnova, Taipei, Taiwan) for $60 \mathrm{~min}$ at room temperature and were then washed 3 times with PBS. Next, the slides were incubated with a horseradish peroxidase/Fab polymer conjugate for a further $30 \mathrm{~min}$. The sites of peroxidase activity were visualized using diaminobenzidine (3, 3'-diaminobenzidine tetrahydrochloride) as the substrate and were counterstained using Mayer's hematoxylin. In the negative control, the primary antibody was omitted and replaced with PBS.

\section{Immunohistochemical scoring system}

The results of phospho-ERK1/2 immunohistochemical staining were analyzed using a semiquantitative scoring system as previously described [21]. The system combined the percentage of immunoreactive 
cells (quantity score) and an estimate of staining intensity (staining intensity score). Each tissue sample was scored according to the quantity and intensity of the staining, with the rating being confirmed by 2 expert pathologists. The intensity score was based on a scale of 0 to 3 as follows $0=$ no staining, $1=$ weak staining, $2=$ moderate staining, and $3=$ strong staining. We subdivided the cytoplasmic as well as nuclear phospho-ERK1/2 immunohistological staining results into low-phospho-ERK1/2 (phospho-ERK1/2 staining 0 and $1+$ ) and high-phospho-ERK1/2 (phospho-ERK1/2 staining $2+$ and $3+$ ) subgroups.
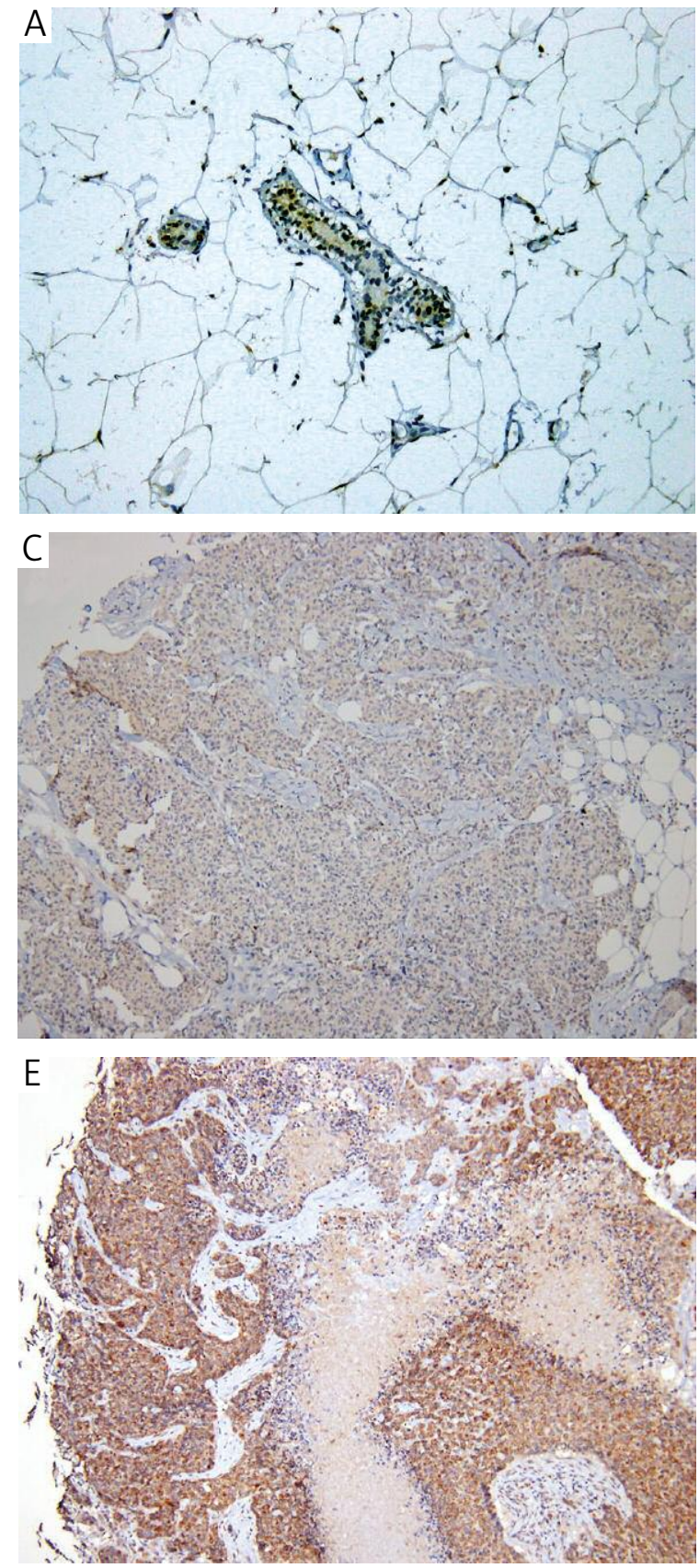

\section{Statistical analysis}

The results of the clinicopathologic variables were tested using Fisher's exact test to ascertain whether differences between the groups were statistically significant. The prognostic ability of each variable was evaluated, including tumor grade, clinical stage, T status, and lymph node metastasis. All analyses were performed using the Statistical Package for Social Sciences (SPSS) version 15.0 (SPSS, Inc, Chicago, IL, USA). A p value less than 0.05 (for a two-tailed test) was considered statistically significant.
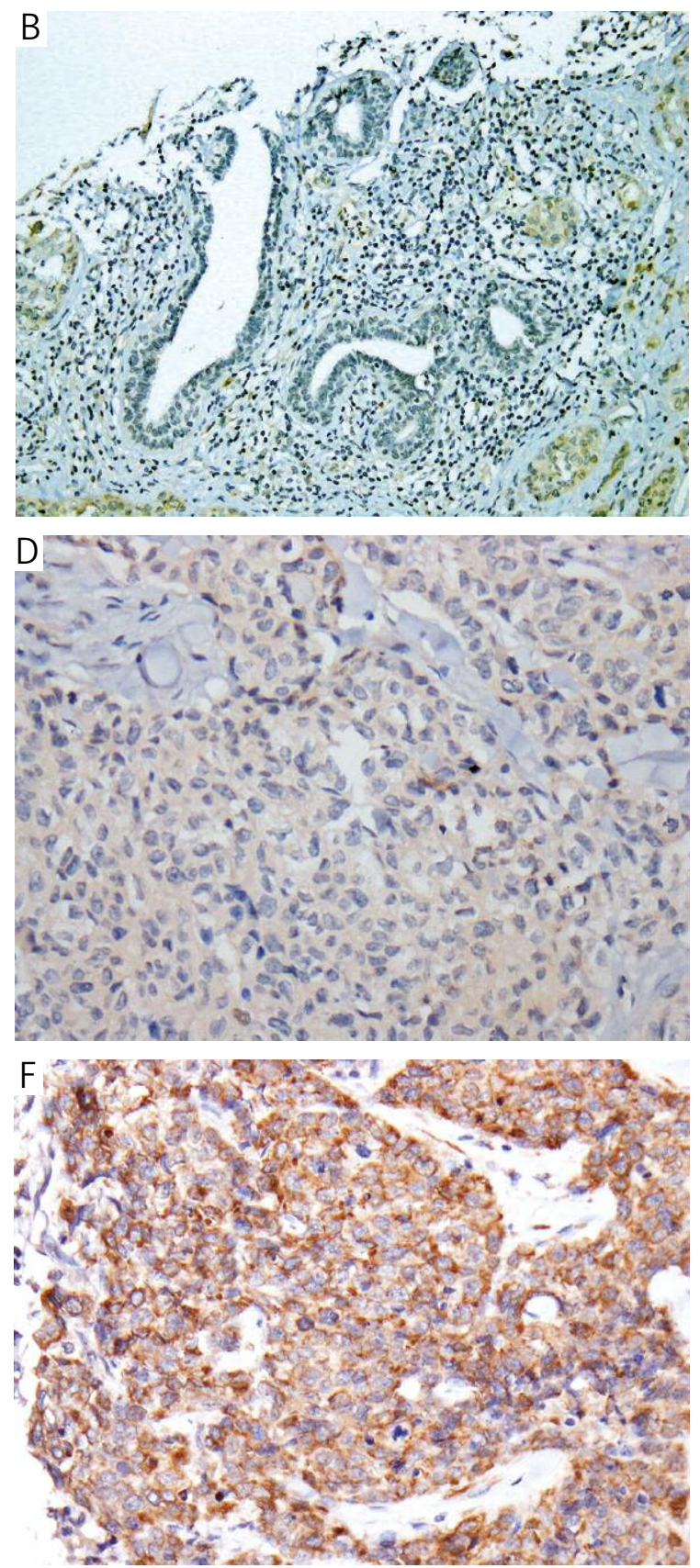

Fig. 1. Representative immunohistochemical images of phospho-ERK1/2 staining in non-tumorous breast tissues and the cytoplasm of breast tumor (A, B). Very weak ( \pm ) staining of phospho-ERK1/2 in non-neoplastic breast tissues (C-F). Cytoplasmic phospho-ERK1/2 staining in breast tumor classified as low expression (C, D), and high expression (E, F). Original magnification: A, C, and E $100 \times$; B, D, and F $400 \times$ 


\section{Results}

We used immunohistochemical analysis to examine the cytoplasmic/nuclear distribution of the activation status of ERK1/2 in breast cancer and the clinical-pathological relationship thereof, using antiphospho-ERK1/2 antibodies (phospho T202/204, G15-B), which are monoclonal antibodies against activated ERK1/2. Phospho-ERK1/2 showed negative or faint staining in non-neoplastic mammary tissues (Fig. 1). In the breast tumor, phospho-ERK $1 / 2$ showed a significant and stronger expression pattern than in the non-neoplastic mammary tissues (Figs. 1 and 2).

The staining intensity of phospho-ERK $1 / 2$ in nonneoplastic mammary tissues was used as an internal positive control, and provided a scoring baseline for phospho-ERK $1 / 2$ staining in the breast tumor specimens. In the breast tumor cells, phospho-ERK $1 / 2$ was positively stained in the cytoplasm, nuclei, or both (Figs. 1 and 2). Based on relative staining intensity of phospho-ERK $1 / 2$ in the cytoplasm and nuclei of tumor cells, we categorized phospho-ERK1/2 expression in the tumor as low-positive $(0,1+)$ and high-positive $(2+, 3+)$ (Figs. 1 and 2). The results showed that 82 cases $(78.1 \%, 82 / 105)$ of the cancers displayed low (weak)
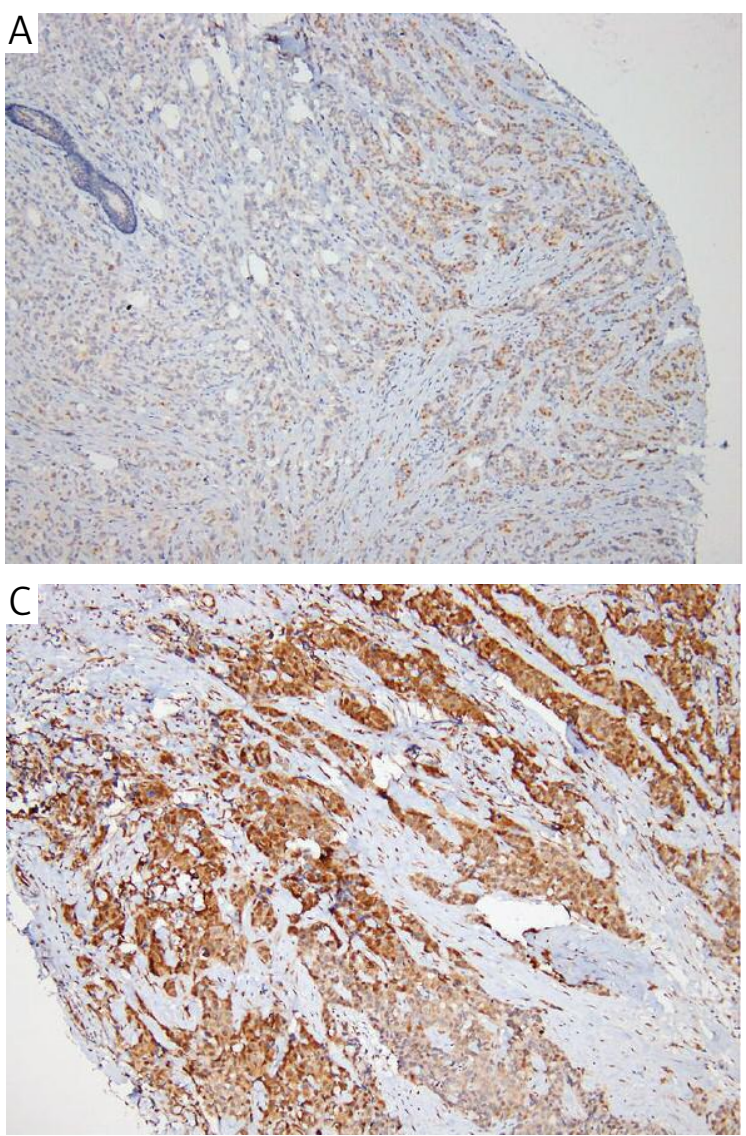

cytoplasmic phospho-ERK1/2 staining, and 23 cases $(21.9 \%, 23 / 105)$ showed high (moderate to strong) cytoplasmic phospho-ERK staining in the breast cancer specimens (Table I). The nuclear phospho-ERK1/2 immunoreactivity showed low (weak) staining in 92 cases $(87.6 \%, 92 / 105)$ and high (moderate to strong) staining in 13 cases $(12.4 \%, 13 / 105)$ of the breast cancer specimens (Table II).

ERK1/2 is expressed in the cytoplasm and activated/phosphorylated ERK1/2 translocates to the nucleus $[6,7]$. We investigated the association between phospho-ERK1/2 cytoplasmic/nuclear distribution and the clinical parameters, including the grade, $\mathrm{T}$ status, stage, and lymph node metastasis of the tumor. Our results showed that no statistically significant association exists between the level of cytoplasmic phospho-ERK1/2 staining and any of the clinical features (Table I). Conversely, the results showed a significant correlation between nuclear phospho-ERK $1 / 2$ distribution and the grade, $\mathrm{T}$ status, and stage of the breast cancer. The statistical results showed that high nuclear phospho-ERK1/2 immunoreactivity in a tumor was significantly correlated with a high grade (poor differentiation, $\mathrm{p}=0.010$ ), high $\mathrm{T}$ status (larger tumor size, $\mathrm{p}=0.033$ ), and an advanced stage $(\mathrm{p}=0.018)$ of the breast cancer
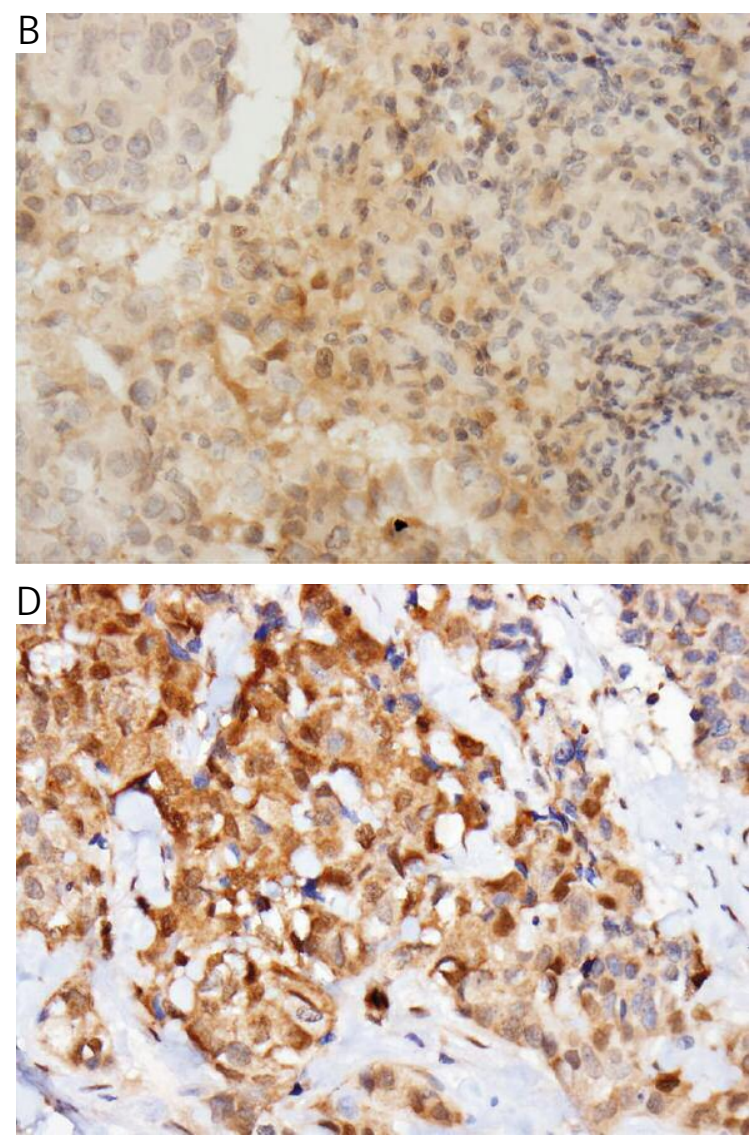

Fig. 2. Representative immunohistochemical images of nuclear phospho-ERK1/2 staining in breast tumor. Breast tumor samples were classified as low $(\mathrm{A}, \mathrm{B})$ and high $(\mathrm{C}, \mathrm{D})$ for nuclear phospho-ERK1/2 staining. Original magnification: $\mathrm{A}, \mathrm{C}-100 \times ; \mathrm{B}, \mathrm{D}-400 \times$ 
Table I. Cytoplasmic phospho-ERK1/2 staining and clinical parameters in breast cancer

\begin{tabular}{|c|c|c|c|c|}
\hline & \multicolumn{2}{|c|}{ CYTOPLASMIC PHOSPHO-ERK1/2 } & \multirow[t]{2}{*}{ Total $(\mathrm{N}=105)$} & \multirow[t]{2}{*}{ P VALUE } \\
\hline & LOW & HIGH & & \\
\hline \multicolumn{5}{|l|}{ Grade } \\
\hline well/moderate & $56(81.2)$ & $13(18.80)$ & 69 & \multirow[t]{2}{*}{0.293} \\
\hline poor & $26(72.2)$ & $10(27.8)$ & 36 & \\
\hline \multicolumn{4}{|l|}{ T status } & \multirow{3}{*}{0.177} \\
\hline $\mathrm{T} 1, \mathrm{~T} 2$ & $52(82.5)$ & $11(17.5)$ & 63 & \\
\hline $\mathrm{T} 3, \mathrm{~T} 4$ & $30(71.4)$ & $12(28.6)$ & 42 & \\
\hline \multicolumn{5}{|l|}{ Stage } \\
\hline $\mathrm{I}$ & $0(0)$ & $0(0)$ & & \multirow{4}{*}{0.115} \\
\hline II & $25(89.3)$ & $3(10.7)$ & 28 & \\
\hline III & $57(74)$ & $20(26)$ & 77 & \\
\hline IV & $0(0)$ & $0(0)$ & & \\
\hline \multicolumn{5}{|c|}{ Lymph node metastasis } \\
\hline no & $4(66.7)$ & $2(33.3)$ & 6 & \multirow[t]{2}{*}{0.610} \\
\hline yes & $78(78.8)$ & $21(21.2)$ & 99 & \\
\hline \multicolumn{5}{|l|}{ Survival } \\
\hline$\leq 2$ years & $16(76.2)$ & $5(23.8)$ & 21 & \multirow[t]{2}{*}{0.813} \\
\hline$>2$ years & $66(78.6)$ & $18(21.4)$ & 84 & \\
\hline
\end{tabular}

Data are shown as number of cases (\%).

Table II. Nuclear phospho-ERK1/2 staining and clinical parameters in breast cancer

\begin{tabular}{|c|c|c|c|c|}
\hline & \multicolumn{2}{|c|}{ NUCLEAR PHOSPHO-ERK1/2 } & \multirow[t]{2}{*}{ TOTAL $(\mathrm{N}=105)$} & \multirow[t]{2}{*}{ P VALUE } \\
\hline & LOW & HIGH & & \\
\hline \multicolumn{5}{|l|}{ Grade } \\
\hline well/moderate & $65(94.2)$ & $4(5.8)$ & 69 & \multirow[t]{2}{*}{$0.010 *$} \\
\hline poor & $27(75.0)$ & $9(25.0)$ & 36 & \\
\hline \multicolumn{5}{|l|}{ T status } \\
\hline $\mathrm{T} 1, \mathrm{~T} 2$ & $59(93.7)$ & $4(6.3)$ & 63 & \multirow[t]{2}{*}{$0.033 *$} \\
\hline $\mathrm{T} 3, \mathrm{~T} 4$ & $33(78.6)$ & $9(21.4)$ & 42 & \\
\hline \multicolumn{5}{|l|}{ Stage } \\
\hline $\mathrm{I}$ & $0(0)$ & $0(0)$ & & \\
\hline II & $28(100)$ & $0(0)$ & 28 & \multirow[t]{3}{*}{$0.018 *$} \\
\hline III & $64(83.1)$ & $13(16.9)$ & 77 & \\
\hline IV & $0(0)$ & $0(0)$ & & \\
\hline \multicolumn{5}{|c|}{ Lymph node metastasis } \\
\hline no & $5(83.3)$ & $1(16.7)$ & 6 & \multirow[t]{2}{*}{0.557} \\
\hline yes & $87(87.9)$ & $12(12.1)$ & 99 & \\
\hline \multicolumn{5}{|l|}{ Survival } \\
\hline$\leq 2$ years & $18(85.7)$ & $3(14.3)$ & 21 & \multirow[t]{2}{*}{0.721} \\
\hline$>2$ years & $74(88.1)$ & $10(11.9)$ & 84 & \\
\hline
\end{tabular}

Data are shown as number of cases (\%).

*statistically significant

(Table II). These results indicated that nuclear, but not cytoplasmic, phosphorylated ERK1/2 plays a significant role in the proliferation and the progression of breast cancer.

\section{Discussion}

MAPK signaling plays an important role in cancer progression, and its activation by upstream signals and 
growth factor stimuli results in cytoplasmic ERK1/2 phosphorylation and phosphorylated ERK1/2, which enter the nucleus to modulate gene transcription and the proliferation and invasion of tumor cells $[6,7]$. The role of ERK $1 / 2$ in the proliferation and invasion of breast cancer cells has been extensively studied in vitro. The phosphorylation and activation of ERK $1 / 2$ by growth factors such as estrogen, epidermal growth factor (EGF), platelet-derived growth factor (PDGF), and insulin in breast cancer cells have been reported [22-24]. Additionally, in vitro studies have reported that ERK1/2 activation was related to the proliferation and motility of breast cancer cells $[15,16]$. Nevertheless, a previous clinical-pathological study by Milde-Langosch et al. of 148 breast cancer samples reported that no positive relationship existed between the activation of ERK1/2 and the progression of breast cancer [17]. The anti-phospho-ERK1/2 monoclonal antibody (Thr202/Tyr204, clone E10, Cell Signaling Technology) that they used did not present any concerns in their study, since that antibody was previously used successfully to assay phosphorylated ERK $1 / 2$ in paraffinembedded tissue samples [25]. ERK1/2 is expressed in the cytoplasm and activated/phosphorylated ERK1/2 translocates to the nucleus to modulate gene transcription and the proliferation and invasion of tumor cells $[6,7]$. In their study, the cytoplasmic and nuclear distribution of the phosphorylated ERK1/2 and their clinical-pathological correlation was not analyzed, and they found no association between ERK1/2 activation and the proliferation and invasion of mammary carcinomas [17]. Comparatively, our study was based on the analysis of the cytoplasmic and nuclear distribution of the phosphorylated ERK1/2 and the clinical-pathological correlation between the two; this may explain the difference in the results of the two studies. However, because ERK1/2 is expressed in the cytoplasm and activated ERK1/2 is distributed in the nuclei of cells to modulate gene transcription and the proliferation and invasion of tumor cells, we propose that activated ERK $1 / 2$ is associated with the proliferation and invasion of breast cancer, and that our results have identified the role of activated ERK1/2 in the progression of breast cancer.

The use of biomarkers is a valuable aid to the diagnosis of breast cancer, as well as the assessment of the patient's prognosis, and facilitates decision-making for treatment [28-30]. In this study, we showed that phospho-ERK1/2 produced negative or faint staining in non-neoplastic mammary tissues, and phosphoERK $1 / 2$ showed a significant and stronger expression in the breast tumor (Figs. 1 and 2). Thus, immunohistological analysis of phosphorylated ERK1/2 in breast tumors might be useful ancillary tool for the diagnosis of breast cancer. Our study also showed that high nuclear phosphorylated ERK1/2 expression in a breast tumor was associated with poor differentiation (high grade), larger tumor size (high $\mathrm{T}$ status), and an advanced stage of breast cancer (Table II). Therefore, the nuclear localization of phosphorylated ERK in a breast cancer tumor may indicate aggressive tumor behavior and may constitute an independent prognostic factor. Breast cancer patients who show high nuclear phosphorylated ERK expression in the tumor may require close follow-up to ensure early and adequate therapy.

The authors declare no conflict of interest.

This study was supported by a grant from the National Science Council (NSC 101-2314-B-038-038) and a grant from the Chi-Mei Medical Center, Tainan, Taiwan (98CM-TMU-01-3).

\section{References}

1. Tran P, Fentiman IS. Better treatment for breast cancer in older patients. Expert Rev Anticancer Ther 2009; 9: 1081-1090.

2. Janni W, Hepp P, Nestle-Kraemling C, et al. Treatment of pregnancy-associated breast cancer. Expert Opin Pharmacother 2009; 10: 2259-2267.

3. Aapro M, Monfardini S, Jirillo A, et al. Management of primary and advanced breast cancer in older unfit patients (medical treatment). Cancer Treat Rev 2009; 35: 503-508.

4. Vodermaier A. Breast cancer treatment and cognitive function: the current state of evidence, underlying mechanisms and potential treatments. Womens Health (Lond Engl) 2009; 5: 503-516.

5. Olszewski WP, Chmielik E, Ryś J. Pathomorphologic patient selection for systemic therapy. Pol J Pathol 2011; 62 (4 Suppl 4): 28-33.

6. Robinson MJ, Cobb MH. Mitogen-activated protein kinase pathways. Curr Opin Cell Biol 1997; 9: 180-186.

7. Chen RH, Sarnecki C, Blenis J. Nuclear localization and regulation of erk- and rsk-encoded protein kinases. Mol Cell Biol 1992; 12: $915-927$.

8. Smith ER, Cai KQ, Smedberg JL, et al. Nuclear entry of activated MAPK is restricted in primary ovarian and mammary epithelial cells. PLoS One 2010; 5: e9295.

9. Hoshino R, Chatani Y, Yamori T, et al. Constitutive activation of the 41-/43-kDa mitogen-activated protein kinase signaling pathway in human tumors. Oncogene 1999; 18: 813-822.

10. Wójcik P, Okoń K, Osuch C, et al. BRAF mutations in sporadic colorectal carcinoma from polish patients. Pol J Pathol 2010; 61: 23-26.

11. Santen RJ, Song RX, McPherson R, et al. The role of mitogenactivated protein (MAP) kinase in breast cancer. J Steroid Biochem Mol Biol 2002; 80: 239-256.

12. Salh B, Marotta A, Matthewson C, et al. Investigation of the MekMAP kinase-Rsk pathway in human breast cancer. Anticancer Res 1999; 19: 731-740.

13. Bartholomeusz C, Gonzalez-Angulo AM, Liu P, et al. High ERK protein expression levels correlate with shorter survival in triplenegative breast cancer patients. Oncologist 2012; 17: 766-774.

14. Wang Z, Wang S, Zhu F, et al. Expression of extracellular signal-regulated kinase and its relationship with clinicopathological characteristics of breast cancer. Zhonghua Zhong Liu Za Zhi 2002; 24: 360-363.

15. Seddighzadeh M, Zhou JN, Kronenwett U, et al. ERK signalling in metastatic human MDA-MB231 breast carcinoma cells is adapted to obtain high urokinase expression and rapid cell proliferation. Clin Exp Metast 1999; 17: 649-654.

16. Krueger JS, Keshamouni VG, Atanashkova N, et al. Temporal and quantitative regulation of mitogen-activated protein ki- 
nase (MAPK) modulates cell motility and invasion. Oncogene 2001; 20: 4209-4218.

17. Milde-Langosch K, Bamberger AM, Rieck G, et al. Expression and prognostic relevance of activated extracellular-regulated kinases (ERK1/2) in breast cancer. Br J Cancer 2005; 92: 2206-2215.

18. Glading A, Koziol JA, Krueger J, et al. PEA-15 inhibits tumor cell invasion by binding to extracellular signal-regulated kinase 1/2. Cancer Res 2007; 67: 1536-1544.

19. American Joint Committee on Cancer Staging Manual. Edge SB, Byrd DR, Compton CC, et al. (eds.). $7^{\text {th }}$ ed. Springer, New York 2009.

20. Tai CJ, Chang CC, Jiang MC, et al. Clinical-pathological correlation of K-Ras mutation and ERK phosphorylation in colorectal cancer. Pol J Pathol 2012; 63: 93-100.

21. Tai CJ, Lee $\mathrm{CH}$, Chen HC, et al. High nuclear expression of phosphorylated ERK in tumor cells in colorectal glands is associated with poor outcome in colorectal cancer. Ann Diagn Pathol 2013; 17: 165-171.

22. Collins P, Webb C. Estrogen hits the surface. Nat Med 1999; 5: 1130-1131.

23. Griffiths MR, Black EJ, Culbert AA, et al. Insulin-stimulated expression of c-fos, fral and c-jun accompanies the activation of the activator protein-1 (AP-1) transcriptional complex. Biochem J 1998; 335: 19-26.

24. Whitmarsh AJ, Davis RJ. Transcription factor AP-1 regulation by mitogen-activated protein kinase signal transduction pathways. J Mol Med 1996; 74: 589-607.

25. Adeyinka A, Nui Y, Cherlet T, et al. Activated mitogen-activated protein kinase expression during human breast tumorigenesis and breast cancer progression. Clin Cancer Res 2002; 8: $1747-1753$.

26. Al-Allak A, Lewis PD, Bertelli G. Decision-making tools to assist prognosis and treatment choices in early breast cancer: a review. Expert Rev Anticancer Ther 2012; 12: 1033-1043.

27. Oakman C, Santarpia L, Di Leo A. Breast cancer assessment tools and optimizing adjuvant therapy. Nat Rev Clin Oncol 2010; 7: 725-732.

28. Weigel MT, Dowsett M. Current and emerging biomarkers in breast cancer: prognosis and prediction. Endocr Relat Cancer 2010; 17: R245-262.

29. Hodorowicz-Zaniewska D, Kibil W, Małek A, et al. Evaluation of serum concentrations of vascular endothelial growth factor (VEGF) in breast cancer patients. Pol J Pathol 2012; 63: 255-260.

30. Adamczyk A, Niemiec J, Ambicka A, et al. Expression of $\mathrm{ER} / \mathrm{PR} / \mathrm{HER} 2$, basal markers and adhesion molecules in primary breast cancer and in lymph nodes metastases: a comparative immunohistochemical analysis. Pol J Pathol 2012; 63: 228-234.

\section{Address for correspondence}

Cheng-Jeng Tai, MD

Department of Internal Medicine

Taipei Medical University Hospital

No. 250, Wu-Hsing St.

Taipei 11031, Taiwan

tel. 886-2-27372181

fax 886-2-2736-3051

e-mail: cjtai@tmu.edu.tw 